\title{
A Predictive Distribution Model for Cooperative Braking System of an Electric Vehicle
}

\author{
Hongqiang Guo, ${ }^{1}$ Hongwen He, ${ }^{1}$ and Xuelian Xiao ${ }^{2}$ \\ ${ }^{1}$ National Engineering Laboratory for Electric Vehicles, Beijing Institute of Technology, Beijing 100081, China \\ ${ }^{2}$ China North Vehicle Research Institute, Beijing 100072, China \\ Correspondence should be addressed to Hongwen He; hwhebit@bit.edu.cn
}

Received 10 October 2013; Accepted 19 December 2013; Published 9 February 2014

Academic Editor: Wudhichai Assawinchaichote

Copyright (c) 2014 Hongqiang Guo et al. This is an open access article distributed under the Creative Commons Attribution License, which permits unrestricted use, distribution, and reproduction in any medium, provided the original work is properly cited.

\begin{abstract}
A predictive distribution model for a series cooperative braking system of an electric vehicle is proposed, which can solve the real-time problem of the optimum braking force distribution. To get the predictive distribution model, firstly three disciplines of the maximum regenerative energy recovery capability, the maximum generating efficiency and the optimum braking stability are considered, then an off-line process optimization stream is designed, particularly the optimal Latin hypercube design (Opt LHD) method and radial basis function neural network (RBFNN) are utilized. In order to decouple the variables between different disciplines, a concurrent subspace design (CSD) algorithm is suggested. The established predictive distribution model is verified in a dynamic simulation. The off-line optimization results show that the proposed process optimization stream can improve the regenerative energy recovery efficiency, and optimize the braking stability simultaneously. Further simulation tests demonstrate that the predictive distribution model can achieve high prediction accuracy and is very beneficial for the cooperative braking system.
\end{abstract}

\section{Introduction}

As a core technology in electric vehicles, cooperative braking with regenerative braking and mechanical braking can not only improve the fuel efficiency but also maintain a satisfying braking stability, as studied in the literature [1-3]. For a cooperative braking system, two braking scenarios are presently studied. The first one is normal deceleration process, within which the braking force distribution strategy is usually studied. The second one is emergency braking process, within which the antilock braking system (ABS) is required to work together with the regenerative braking simultaneously [4].

Generally, when the vehicle is decelerating, the normal deceleration process happens in most cases, which indicate that the braking force distribution strategy is vital for the cooperative braking system. With respect to this, how to maximize the regenerative energy recovery efficiency under the premise of braking stability is the focus of traditional study. Many researchers have made many efforts to develop different distribution strategies. Based on the braking theory, the references [5-11] presented a distribution strategy, in which the braking stability was taken as a precondition, and the maximum regenerative energy recovery efficiency was regarded as the target. If the generators met the required braking force, the braking force was afforded by generators, otherwise, hydraulic brakes compensated the rest braking force. The reference [11] considered the low generating efficiency of generators, and a similar distribution strategy was proposed according to the ECE braking regulation. The references $[12,13]$ proposed an optimization method in which braking stability was regarded as the constraint and the maximum regenerative energy recovery efficiency was designed as the target. Generally, the strategies described above focused mainly on the objective of the regenerative energy recovery efficiency. However, the braking stability is also very important. Braking strategies which can maximize the regenerative energy recovery efficiency and optimize the braking stability objectives simultaneously are a key technology in cooperative braking systems. Additionally, the rapidity of a real-time control may affect the performance of the cooperative braking 


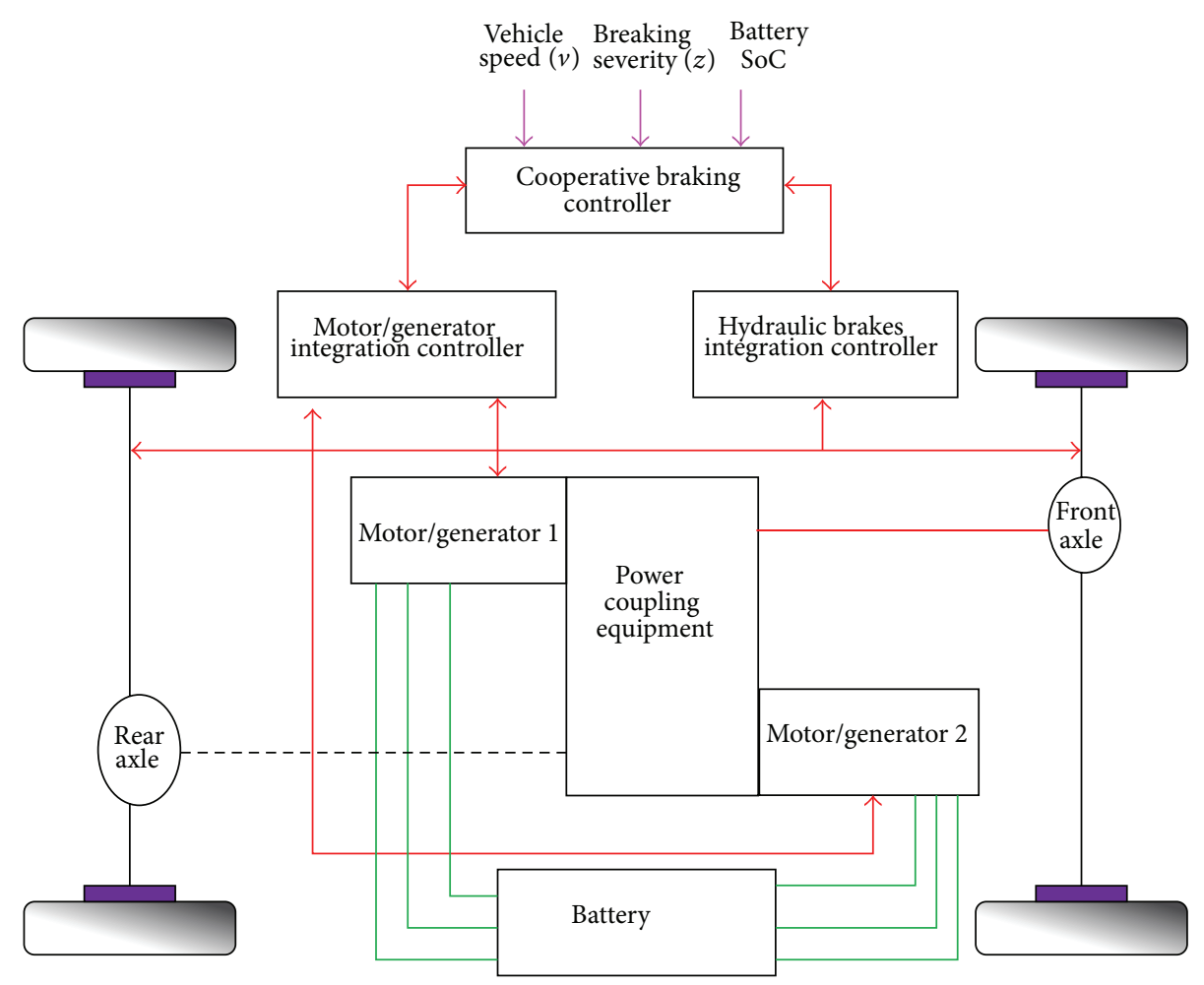

FIgURE 1: The cooperative braking system of the electric vehicle.

systems, so good performance of the real-time control is also important.

The cooperative braking systems can be classified into parallel and series types [14]. With the parallel system, motor/generators add an additional braking force on the hydraulic braking force, and there will be no coordinate between motor/generators and mechanical hydraulic brakes. Considering the braking stability requirement, the regenerative energy recovery efficiency is usually constrained, but the traditional braking system does not need to be reformed [15]. With the series system, a real-time coordinate between motor/generators and hydraulic brakes is considered. In addition, the total output braking force will follow the required braking force. Since series systems can achieve better regenerative energy recovery efficiency and better braking stability simultaneously, it has been in the spotlight [16-19].

In this paper, a series cooperative braking system with two motor/generators is studied for the normal deceleration process. Different from the traditional methods, a braking force distribution strategy based on a predictive distribution model is proposed. The predictive distribution model is constituted by a predictive model and an additional condition. To get the predictive model, some key technologies have been studied. Firstly, a general mathematical model is established by three disciplines and a braking force analysis. Secondly, an off-line process optimization stream which is constituted by the optimal Latin hypercube design (Opt LHD) and a concurrent subspace design (CSD) method is designed to get an off-line optimization data. Thirdly, a predictive model based on a radial basis function neural network (RBFNN) by the off-line optimization data and the general mathematical model is presented. Finally, the predictive distribution model is verified in a dynamic simulation framework.

\section{The Cooperative Braking Structure}

Figure 1 shows the cooperative braking system of an electric vehicle. The cooperative braking system is constructed by four hydraulic brakes and two motor/generators. The two motor/generators are connected to a power coupler. Fourwheel drive, front-wheel drive, and rear-wheel drive can be realized according to different working modes of the power coupler. This paper only focuses on the front-wheel drive mode. For motor/generator 1, the coupling ratio is 1.442; for motor/generator 2, the coupling ratio is 2.225. The vehicle speed is $v$, the breaking severity is $z$, and the battery SoC are considered as inputs, and the cooperative braking predictive distribution model is implemented in the cooperative braking controller. When the vehicle decelerates, the motor/generators work as generators, the cooperative braking controller can coordinate the braking force distribution between hydraulic brakes and generators. In addition, based on the charging performance of the battery, SoC is constrained by 0.1-0.8. Considering the constraint of the coupler ration, $v$ is constrained by $10-50 \mathrm{~km} / \mathrm{h}$. Since a normal deceleration process is studied, $z$ is constrained by $0.01-0.4$. The scopes of SoC, $v$, and $z$ constitute the continuous design space in this paper. 


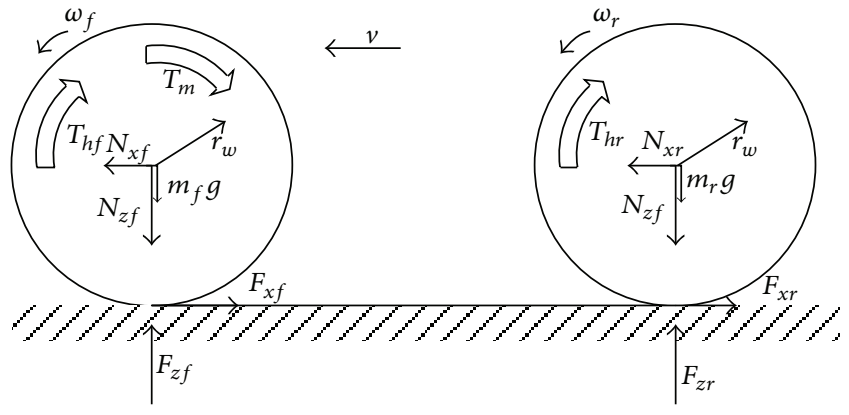

FIgURE 2: The force diagram of the cooperative braking system.

\section{The Cooperative Braking Mathematical Model}

3.1. General Mathematical Model. A force diagram of the cooperative braking system is shown in Figure 2.

In Figure 2, $T_{m}$ denotes the total regenerative braking torque, which is enforced on the front wheels. $T_{h f}, T_{h r}$ are hydraulic braking torques which are applied the on front and rear wheels, respectively; $F_{x f}, F_{x r}$ denote the road braking forces the on front and rear wheels, respectively; $F_{z f}, F_{z r}$ denote the road normal reaction forces the on front and rear wheels, respectively; $r_{w}$ denotes the radius of the wheels; $\omega_{f}$, $\omega_{r}$ denote the angular velocities of the front and rear wheels, respectively; $N_{x f}, N_{x r}$ denote the thrusts of the front and rear wheel axles, respectively; $m_{f} \mathcal{g}, m_{r} g$ denote the gravities of the front and rear wheel axles, respectively.

A general cooperative braking mathematical model is established to induce $T_{m}, T_{h f}$, and $T_{h r}$. The sum of the hydraulic braking torques and the total regenerative braking torque should be equal to the required braking torque. Consider the following:

$$
T_{h f}+T_{m}+T_{h r}=T_{r m}
$$

where $T_{r m}$ is the required braking torque, which is given by (2).

$$
T_{r m}=m g r_{w} z
$$

where $m$ is the total mass of the vehicle.

When the vehicle decelerates, the generators will work simultaneously.

$$
T_{m 1}+T_{m 2}=T_{m}
$$

where $T_{m 1}, T_{m 2}$ denote the regenerative braking torques of the generator 1 and the generator 2 , respectively.

According to (1)-(3), $T_{r m}$ can be viewed as a known variable, however, $T_{m}, T_{h f}, T_{h r}, T_{m 1}$, and $T_{m 2}$ are unknown variables, and only two equations which are (1) and (3) are presented, so another three balance equation should be required.
Define

$$
\begin{gathered}
\frac{T_{m 1}}{T_{m}}=\lambda, \\
\frac{T_{h f}+T_{m}}{T_{r m}}=\beta, \\
\frac{T_{h f}}{T_{h f}+T_{h r}}=\alpha,
\end{gathered}
$$

where $\lambda$ is the regenerative braking torque distribution coefficient.

Usually, $\lambda$ is regarded as a known variable; however, in the optimization mathematic model, it is an unknown variable, and will be varied from 0 to 1 . If $\lambda=0$, it means that the whole regenerative braking torque is only provided by generator 2; similarly, if $\lambda=1$, it means that the whole regenerative braking torque is only provided by generator 1 ; additionally, if $\lambda \in(0,1)$, it means that the whole regenerative braking torque is provided by generator 1 and generator 2 simultaneously. It is significant to define this parameter, for generators; the generating efficiency will be different under different total regenerative braking torques and motor speeds, so it is necessary to find an optimum distribution coefficient to maximize the generating efficiency between two generators. $\beta$ is the braking torque distribution coefficient between front and rear wheels. The same as $\lambda$, herein, it is a known variable, but in optimization mathematic model it is an unknown variable and will be varied by the requirement of the braking regulation and braking stability. $\alpha$ is the braking torque distribution coefficient between hydraulic brakes. It can coordinate the hydraulic brakes to meet the objectives of optimization, and varies from 0 to 1 in optimization mathematical model. If $\alpha=0$, it means that only the front axle hydraulic brakes work; if $\alpha=1$ it means that only rear axle hydraulic brakes work; if $\alpha \in(0,1)$, it means that all the hydraulic brakes will work simultaneously.

According to the above equations, $T_{m}, T_{h f}, T_{h r}, T_{m 1}$ and $T_{m 2}$ can be formulated as follows:

$$
\begin{aligned}
& T_{m}=\frac{\beta-\alpha}{1-\alpha} T_{r m}, \\
& T_{h f}=\frac{\alpha(1-\beta)}{1-\alpha} T_{r m}, \\
& T_{h r}=(1-\beta) T_{r m}, \\
& T_{m 1}=\lambda T_{m}, \\
& T_{m 2}=(1-\lambda) T_{m} .
\end{aligned}
$$

3.2. Optimization Mathematical Model. Three disciplines are proposed in this study. The first one is the maximum regenerative energy recovery capability discipline, which the total regenerative braking torque should be close to the optimum charging torque. The second one is the maximum generating efficiency discipline, in which the charging torque efficiency will be maximized under the given total regenerative braking torque of the first discipline. The third one is the optimum 
braking stability discipline, in which can be classified into four levels as follows.

(1) No wheels are locked, each wheel maintains rolling and sliding state simultaneously, with this state, ABS is usually used. In this study, no ABS is equipped.

(2) No wheels are locked earlier, if locked, all the wheels will be locked simultaneously. In this case, vehicle will keep the optimum braking stability. It is also the optimum braking stability objective in this study, which the braking force distribution coefficient should be close to it.

(3) Front wheels will be locked earlier than rear wheels. In this case, the steering capability will be lost but still is a stability state. In this study, it is viewed as a precondition for braking stability.

(4) Rear wheels will be locked earlier than front wheels. In this case, rear wheels will spin and will be in an instability state. In this study, it is considered as an infeasible region.

A concurrent subspace design (CSD) is performed here and its optimization mathematical model can be set up as follows.

(a) The Maximum Regenerative Energy Recovery Capability Discipline. Consider the following:

Find: $\quad \alpha, \beta$

Minimize: $f_{1}(\alpha, \beta)=\left(\frac{T_{m}-T_{\mathrm{opt}}}{T_{\mathrm{opt}}}\right)^{2}$

Satisfy: $\quad T_{m}+T_{h f}<T_{f}$,

$T_{h r}<T_{r}$

$c=\beta-\alpha>0$,

$b_{1}<\beta<b_{2}$,

$0<\alpha<1$

$$
T_{m}<T_{\text {opt }}
$$

Output: $T_{m}$,

where $c$ is an auxiliary constraint, which can ensure $T_{m}>0$; $T_{f}, T_{r}$ are the maximum braking torques which are subjected to a dry pavement; and $T_{\text {opt }}$ denotes the optimum charging torque, which can be expressed as

$$
T_{\text {opt }}=\min \left[\max \left(T_{\text {mot_brake }}\right), \max \left(T_{\text {battery_charge }}\right)\right],
$$

where $\max \left(T_{\text {mot_brake }}\right)$ is the maximum regenerative braking torque; $\max \left(T_{\text {battery_charge }}\right)$ is the maximum rechargeable torque; and $b_{1}, b_{2}$ denote the lower and upper boundary conditions of the braking stability, respectively, which are defined according to the braking regulation of the ZBT24007-1989 in (8) and the braking stability precondition in (9) as follows:

$$
\begin{gathered}
\beta \geq \frac{b+z h_{g}}{L}, \quad 0 \leq z \leq 0.3 \\
\frac{(z-0.08)\left(b+z h_{g}\right)}{z L}<\beta \leq \frac{(z+0.08)\left(b+z h_{g}\right)}{z L}, \\
\beta \geq 1-\frac{(z+0.25) L}{0.74\left(a-z h_{g}\right) z}, \quad 0.3 \leq z \leq 0.8 \\
1-\frac{\left(a-z h_{g}\right)((z-0.1) / 0.85+0.2)}{L z} \\
\leq \beta \leq \frac{\left(b+z h_{g}\right)((z-0.1) / 0.85+0.2)}{L z}, \quad 0.2 \leq z \leq 0.8 \\
\beta \geq \frac{b+z h_{g}}{L}, \quad 0 \leq z \leq 0.8
\end{gathered}
$$

where $a, b, L$, and $h_{g}$ denote the front wheelbase, rear wheelbase, wheelbase, and the height of vehicle body mass center respectively.

(b) The Maximum Generating Efficiency Discipline. Consider the following:

Given: $\quad T_{m}, n$

Find: $\quad \lambda$

Minimize: $f_{2}(\lambda)=\frac{1}{f_{\text {generator1_efficiency }} f_{\text {generator_efficiency }}}$

Satisfy: $\quad 0 \leq \lambda \leq 1$,

$$
\begin{aligned}
& T_{m 1} \leq T_{\text {out1 }}, \\
& T_{m 2} \leq T_{\text {out } 2}
\end{aligned}
$$

Output: $f_{2}$,

where $n$ is the generator's speed; $f_{\text {generatorl_efficiency }}$ and $f_{\text {generator2_efficiency }}$ denote the generator's efficiencies, which can be obtained by an efficiency map in Figure 3; $T_{\text {out1 }}, T_{\text {out } 2}$ are the maximum output regenerative torques which can be got by Figure 4 .

(c) The Optimum Braking Stability Discipline. Generally, if the adhesions between front and rear wheels are equal under any braking severity, the vehicle can get the best braking stability [7]; in this state, the braking force distribution curve is the ideal curve ( $I$ curve). Thus the optimum braking distribution coefficient can be realized as follows:

$$
\beta_{\mathrm{opt}}=\frac{b+z h_{g}}{L} \text {. }
$$




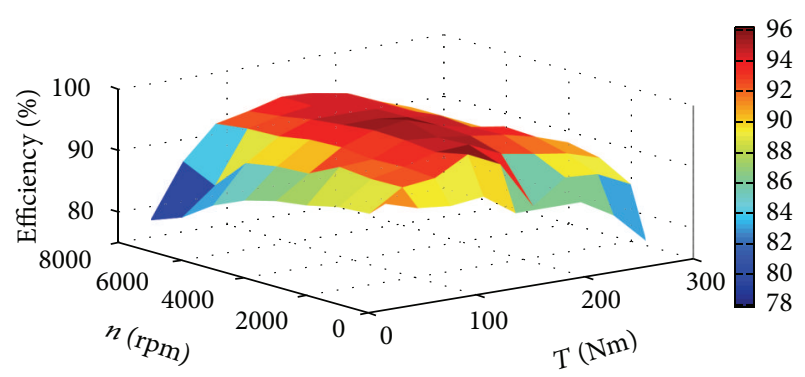

FIgURE 3: The efficiency map of the generators.

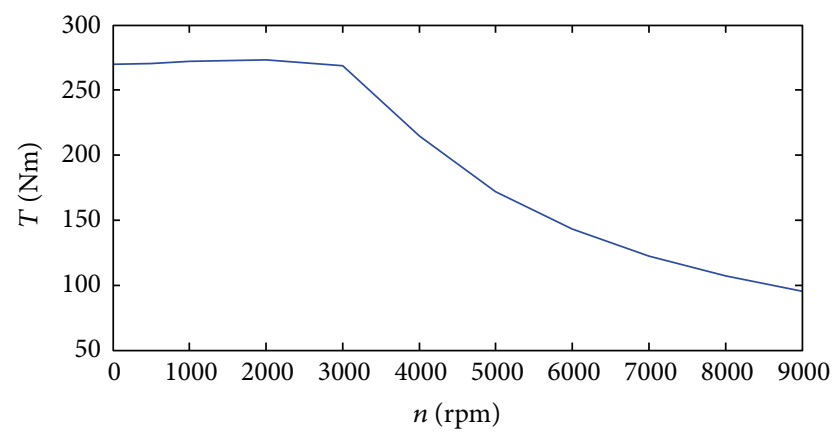

FIGURE 4: The regenerative torque versus speed curve.

Additionally, if $\beta>\left(b+z h_{g}\right) / L$, it means that the adhesion of the front wheels is bigger than the rear wheels, and it is also a stability state.

The optimization mathematical model can be expressed as follows:

$$
\begin{aligned}
& \text { Find: } \quad \beta \\
& \text { Minimize: } f_{3}(\beta)=\left(\frac{\beta-\beta_{\mathrm{opt}}}{\beta_{\mathrm{opt}}}\right)^{2} \\
& \text { Satisfy: } \quad b_{1}<\beta<b_{2} .
\end{aligned}
$$

3.3. Coupling Relationship between Different Disciplines. As can be seen in Figure 5, $\alpha, \beta$, and $\lambda$ are system variables; $\beta$ is the system coupling variable, which can affect discipline 1 and discipline 3 simultaneously; $T_{m}$ is the discipline coupling variable, which is both the output variable of discipline 1 and the input variable of discipline 2 ; and $f_{1}, f_{2}$, and $f_{3}$ are the output variables which are passed to the system.

3.4. Off-Line Process Optimization Stream. As shown in Figure 6, design of experiment (DOE) 1 and another three modules are defined in the stream. DOE 1 is used to discrete the continuous input design space with the optimal Latin hypercube design (Opt LHD) method. For the three modules, we have the following.

(1) Module 1 is the original approximation model module. Since different sampling data correspond to different inputs,

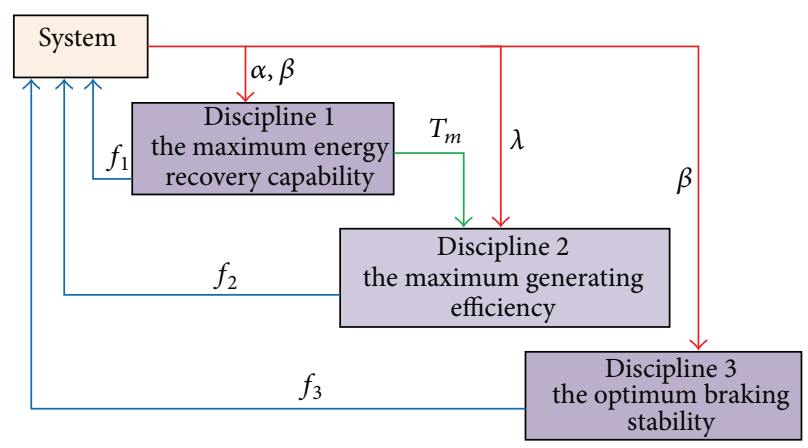

FIGURE 5: The coupling relationship between different disciplines.

in order to ensure the generated sampling data corresponding to the given input parameter, deleting the existing sampling data firstly is necessary in this module (delete the existing sampling point data component). $T_{\mathrm{opt}}, \beta_{\mathrm{opt}}, T_{r m}$, $T_{\text {out } 1}, T_{\text {out } 2}, b_{1}, b_{2}, T_{f}$, and $T_{r}$ can be calculated by the calculation program component. DOE 2 component is used to discrete the design variables with the Opt LHD method. An approximation model with respect to $\widetilde{T}_{m}, \widetilde{T}_{m 1}, \widetilde{T}_{m 2}, \widetilde{T}_{h f}$, $\widetilde{T}_{h r}, \widetilde{f}_{1}, \widetilde{f}_{2}$, and $\widetilde{f}_{3}$ can be built by the approximation model component, which $\widetilde{T}_{m}, \widetilde{T}_{m 1}, \widetilde{T}_{m 2}, \widetilde{T}_{h f}, \widetilde{T}_{h r}, \widetilde{f}_{1}, \widetilde{f}_{2}$, and $\widetilde{f}_{3}$ are the approximate values of $T_{m}, T_{m 1}, T_{m 2}, T_{h f}, T_{h r}, f_{1}, f_{2}$ and $f_{3}$.

(2) Module 2 is the CSD algorithm module, which is constituted by a system optimization layer and three discipline layers. For the discipline layers, no optimization will be carried out according to the CSD algorithm; the only role for them is to add three new sets of sampling points to update the sampling point component in every cycle and accelerate the convergence of the approximation model. Aiming at a specific discipline layer, the approximation values of other disciplines will be provided with the approximation model and the real values of the specific discipline will be obtained with the real mathematic model. According to the update of the sampling points component, a higher-precision approximation model will be obtained by the update approximation model component, based on which the system optimization layer can carry out the optimization task; additionally, the optimization mathematic model is shown in (13). With the iterative calculation of the optimization, the approximation model precision will be higher and higher and the optimization results will be converged sooner.

(3) Module 3 is the predictive model module. The most important task for it is to set up a high-precision predictive model. The input parameters of SoC, $v$, and $z$ are viewed as inputs, and the optimization values of $\alpha, \beta$, and $\lambda$ which are denoted by $\alpha_{\text {optimization }}, \beta_{\text {optimization }}$, and $\lambda_{\text {optimization }}$ are viewed as outputs. A radial basis function neural network (RBFNN) will be generated based on the inputs and outputs. Additionally, a feedback cycle-control strategy is designed. If the precision of the RBFNN meets the requirement, then, end; otherwise, additional sampling points will be added to the DOE 1 component. 


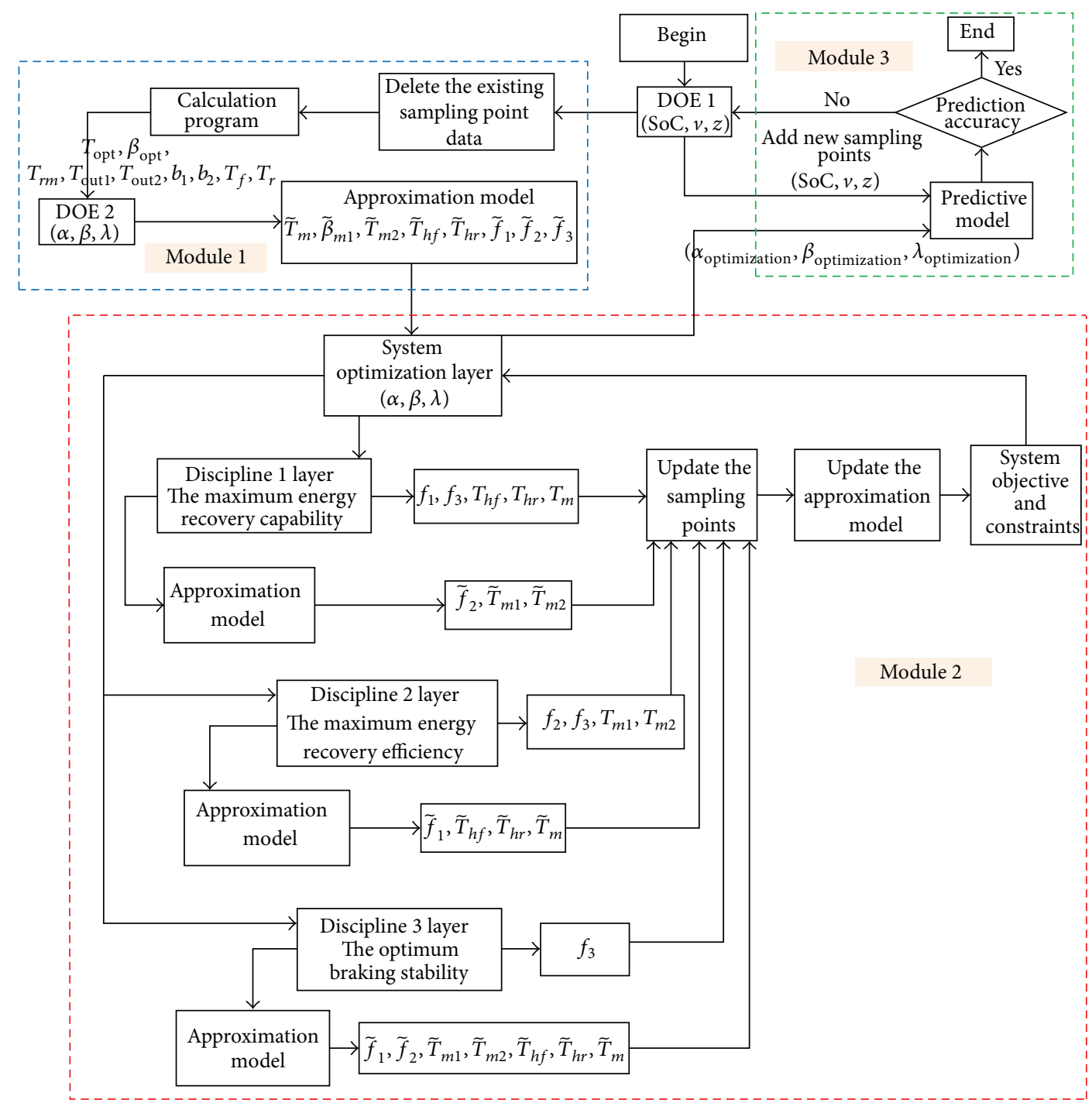

FIgURE 6: The off-line process optimization stream.

Then, the optimization mathematic model is

$\tilde{f}_{1}=\left(\frac{\widetilde{T}_{m}-T_{\mathrm{opt}}}{T_{\mathrm{opt}}}\right)^{2}$

$\tilde{f}_{2}=\frac{1}{\tilde{f}_{\text {generator1_efficiency }} \tilde{f}_{\text {generator2_efficiency }}}$,

$f_{3}=\left(\frac{\beta-\beta_{\mathrm{opt}}}{\beta_{\mathrm{opt}}}\right)^{2}$,

Find $\quad \alpha, \beta, \lambda$

Minimize $F(\alpha, \beta, \lambda)$

$$
=w_{1}\left(\frac{\widetilde{T}_{m}-T_{\mathrm{opt}}}{T_{\mathrm{opt}}}\right)^{2}
$$

$+\frac{w_{2}}{\widetilde{f}_{\text {generator1_efficiency }} \widetilde{f}_{\text {generator2_efficiency }}}$

$+w_{3}\left(\frac{\beta-\beta_{\mathrm{opt}}}{\beta_{\mathrm{opt}}}\right)^{2}$

Satisfy $\quad \widetilde{T}_{m}+\widetilde{T}_{h f}<T_{f}$,

$\widetilde{T}_{h r}<T_{r}$,

$c=\beta-\alpha>0$,

$b_{1}<\beta<b_{2}$,

$0<\alpha<1$

$\widetilde{T}_{m}<T_{\text {opt }}$,

$0 \leq \lambda \leq 1$ 


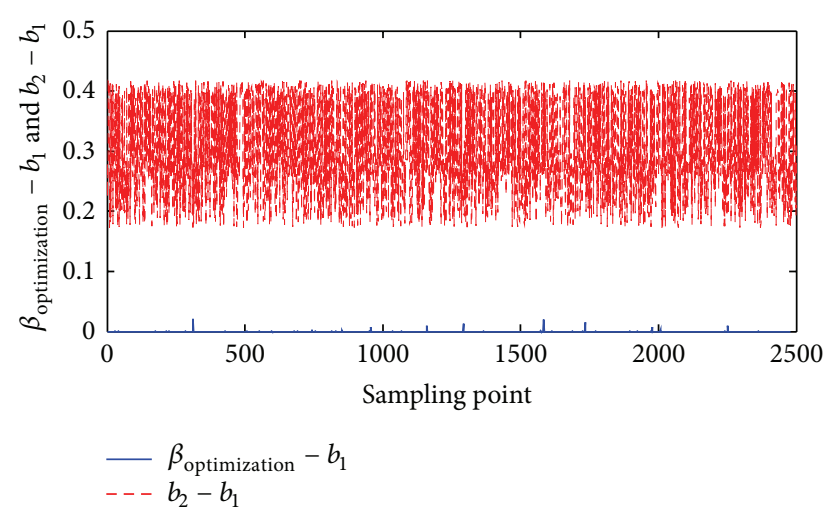

FIGURE 7: The braking force distribution coefficient.

$$
\begin{aligned}
& \widetilde{T}_{m 1} \leq T_{\text {out1 }}, \\
& \widetilde{T}_{m 2} \leq T_{\text {out } 2},
\end{aligned}
$$

where $w_{1}, w_{2}$, and $w_{3}$ are weighting coefficients, in which $w_{1}$ and $w_{3}$ are 1 and $w_{2}$ is 0.01 .

\section{Results and Discussions}

4.1. Off-Line Optimization Results. As shown in Figure 7, $\beta_{\text {optimization }}$ should be bigger than or equal to $\beta_{\text {opt }}$, according to the braking stability requirement. Herein, $\beta_{\text {optimization }}-$ $b_{1}$ is close to 0 but bigger than 0 , and lower than $b_{2}-b_{1}$, which means that the vehicle can always keep a better braking stability.

Define

$$
\begin{aligned}
& \varepsilon=\frac{T_{m_{-} \text {optimization }}-T_{\mathrm{opt}}}{T_{\mathrm{opt}}} 100 \%, \\
& \xi=\frac{T_{m_{-} \text {optimization }} f_{2}-T_{\mathrm{opt}}}{T_{\mathrm{opt}}} 100 \%,
\end{aligned}
$$

where $\varepsilon$ denotes the deviate error between the regenerative braking torque and the ideal charging torque; $\zeta$ denotes the deviate error between the charging torque between actual and ideal; and $T_{m \_ \text {optimization }}$ denotes the optimization value of $T_{m}$.

It can be seen from Figure 8 that the value of $\varepsilon$ is very small and below zero, which means that the total regenerative braking torque always follows $T_{\mathrm{opt}}$. Two generators can coordinate themselves to maximize the generating efficiency. For that, the average value of $\zeta$ is only $20 \%$, and the average generating efficiency of the generators is nearly $80 \%$.

As shown in Figure 9, $\alpha_{\text {optimization }}$ is close to 0 at some sampling points, which means that only the hydraulic braking torque of rear wheels is needed to realized the optimum braking force distribution. Apart from those sampling points, the value of $\alpha_{\text {optimization }}$ is between 0 and 1, which means that all the hydraulic brakes will be worked. Similarly, with respect to $\lambda_{\text {optimization }}$, at some sampling points, $\lambda_{\text {optimization }}$ is close to 1 , which means that with only generator 1 offering the

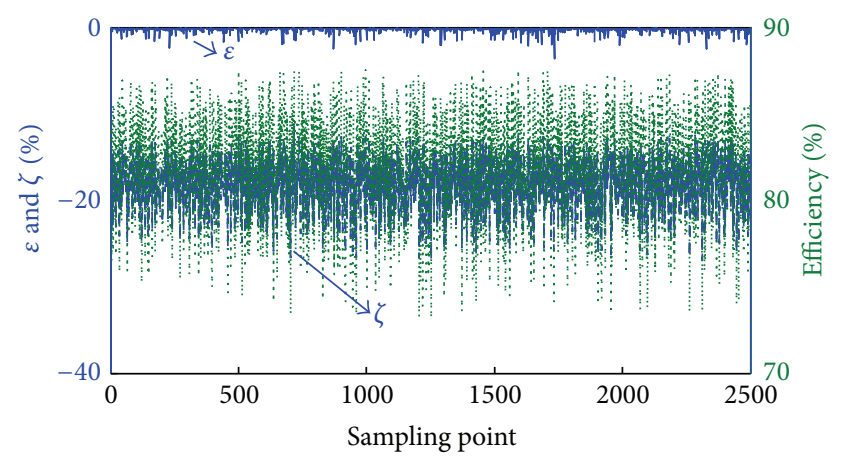

Figure 8: $\varepsilon, \zeta$, and generating efficiency.

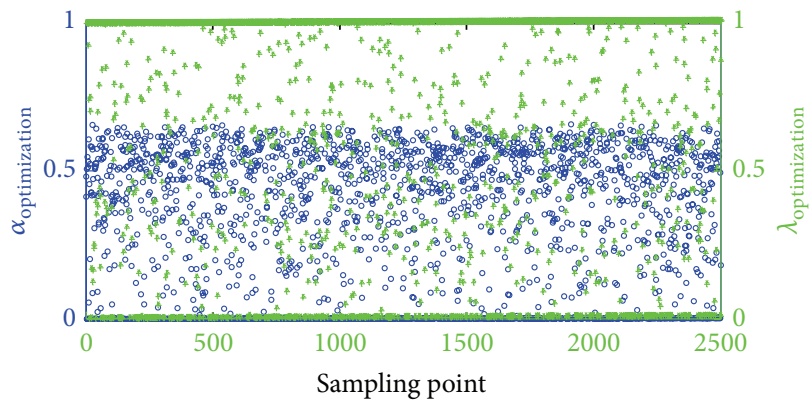

FIGURE 9: $\alpha_{\text {optimization }}$ and $\lambda_{\text {optimization }}$.

total regenerative braking torque, the generating efficiency can be maximized; on the contrary, when $\lambda_{\text {optimization }}$ is close to 0 , it means that with only generator 2 offerring the total regenerative braking torque, the generating efficiency can be maximized. Additionally, when $\lambda_{\text {optimization }}$ is between 0 and 1 , it means that the generating efficiency can be maximized when the two generators work simultaneously.

The above optimization results show that the optimization model with the CSD method can get good results, and lay a good foundation for a further high-presicison predictive model.

4.2. Predictive Distribution Model. Considering the poor real-time control of the optimization, a predictive distribution model together with a predictive model and an additional condition is presented. For the predictive model, the multiple correlation coefficients $\left(R^{2}\right)$ of $\widehat{\alpha}, \widehat{\beta}$, and $\hat{\lambda}$ are $0.95,0.999$, and 0.96045 , respectively. Generally, although $\widehat{\alpha}$, $\widehat{\beta}$, and $\hat{\lambda}$ have a relatively high predictive precision, predictive errors still exist; a relatively small predictive error may cause the predictive parameters to fall into the infeasible region.

To ensure that the predictive distribution model meets the cooperative braking requirement, some key predictive parameters' scope should be verified as follows.

(1) As stated above, $T_{m}>0$ is the precondition to realize the regenerative braking energy recovery. So, $\widehat{\alpha}$ should be lower than $\widehat{\beta}$ based on (5). Figure 10 shows the predictive results of $\widehat{\alpha}-\widehat{\beta}$ which is denoted by $\alpha_{\text {predictive value }}-\beta_{\text {predictive value }}$. It can be seen that 


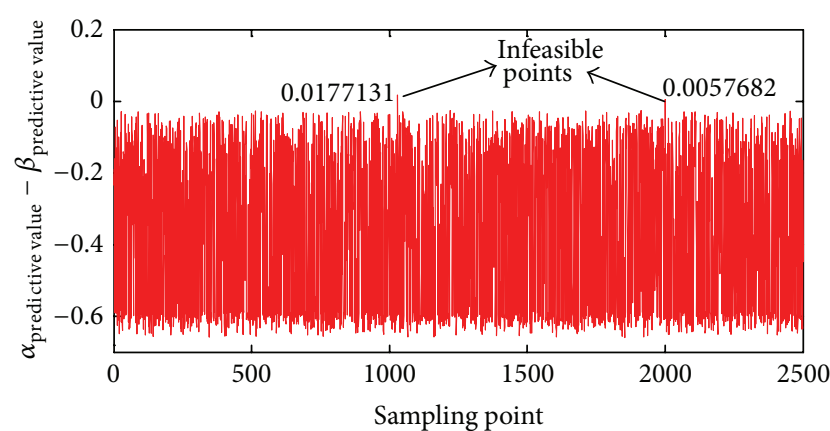

FIGURE 10: The predictive results of $\alpha_{\text {predictive value }}-\beta_{\text {predictive value }}$.

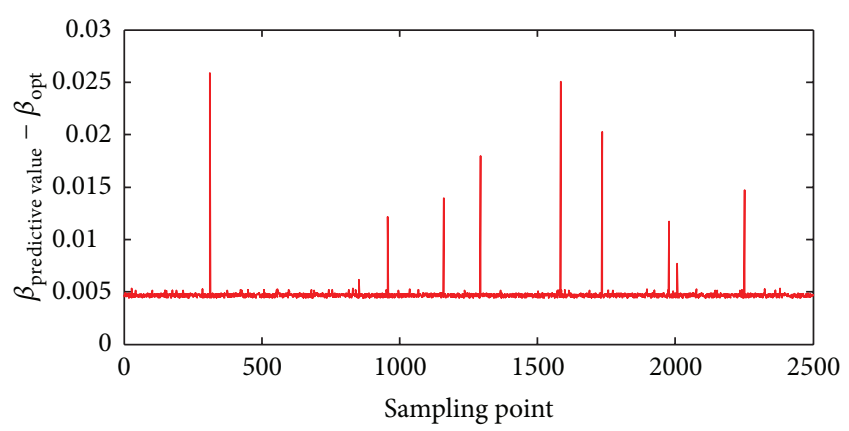

FIGURE 11: The predictive results of $\beta_{\text {predictive value }}-\beta_{\text {opt }}$.

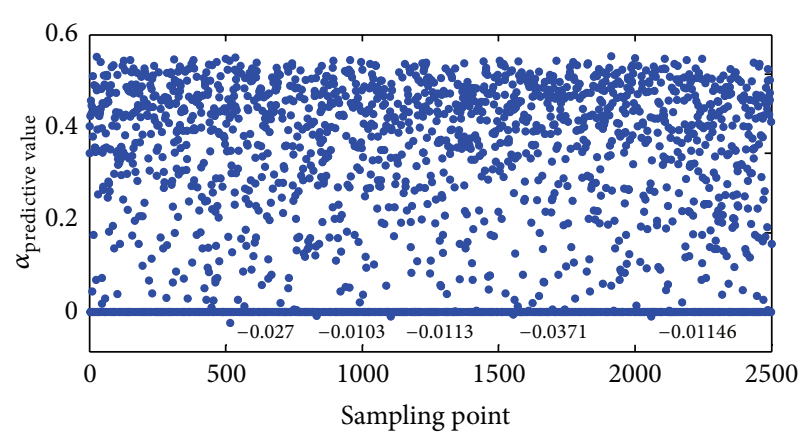

FIGURE 12: The predictive results of $\alpha_{\text {predictive }}$.

an overwhelming majority of the sampling points can meet the requirement of the cooperative braking system except two sampling points.

(2) With respect to $\widehat{\beta}$, it should be bigger than or equal to $\beta_{\text {opt }}$ according to the requirement of braking stability. Figure 11 shows the predictive results of $\widehat{\beta}-\beta_{\mathrm{opt}}$ which is denoted by $\beta_{\text {predictive value }}-\beta_{\text {opt }}$. Obviously, due to the high predictive precision of $\widehat{\beta}$, it can always meets the requirement of braking stability.

(3) With regard to $\widehat{\alpha}$ and $\widehat{\lambda}$. $\widehat{\alpha}$ should not be lower than $0 ; \hat{\lambda}$ should not be lower than 0 and bigger than 1 according to the requirement in (4). Figures 12 and 13 show the predictive values of $\hat{\alpha}$ and $\hat{\lambda}$, which are denoted by $\alpha_{\text {predictive value }}$ and $\lambda_{\text {predictive value. }}$. Both of the

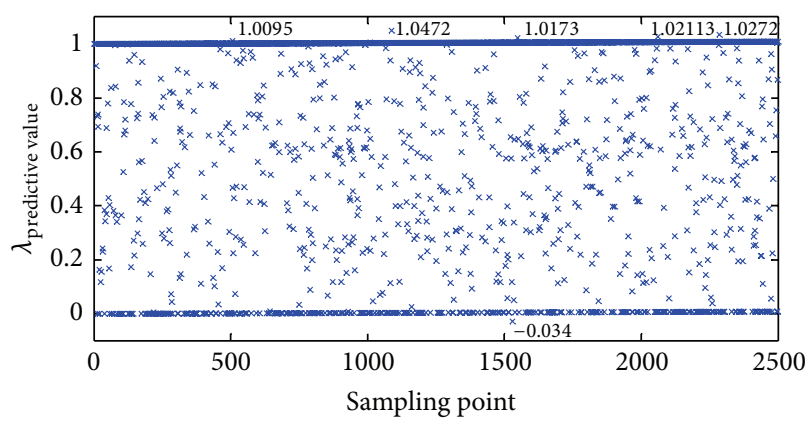

FIGURE 13: The predictive results of $\lambda_{\text {predictive value }}$.

predictive parameters have some infeasible sampling points.

Given the above analysis, an additional condition should be added to the predictive distribution model. For the additional condition, the basic principle is that, if the predictive parameters fall into the infeasible region, the braking torque will be only provided by hydraulic brakes. The predictive distribution model is shown as follows:

$$
\begin{aligned}
& \widehat{T}_{m}=\frac{\widehat{\beta}-\widehat{\alpha}}{1-\widehat{\alpha}} T_{r m}, \\
& \widehat{T}_{h f}=\frac{\widehat{\alpha}(1-\widehat{\beta})}{1-\widehat{\alpha}} T_{r m}, \\
& \widehat{T}_{h r}=(1-\widehat{\beta}) T_{r m}, \\
& \widehat{T}_{m 1}=\hat{\lambda} \widehat{T}_{m}, \\
& \widehat{T}_{m 2}=(1-\widehat{\lambda}) T_{r m}, \\
& \text { Define: } \eta=2.5 \\
& \text { If } \quad \widehat{\alpha}<0\|\hat{\lambda}>1\| \hat{\lambda}<0 \| \widehat{\alpha}-\widehat{\beta}>0, \\
& \quad \widehat{T}_{m 1}=0, \\
& \quad \widehat{T}_{m 2}=0, \\
& \quad \widehat{T}_{h f}=\frac{\eta T_{r m}}{(1+\eta)}, \\
& \widehat{T}_{h r}=\frac{T_{r m}}{(1+\eta)},
\end{aligned}
$$

End,

where $\widehat{\alpha}, \widehat{\beta}$, and $\hat{\lambda}$ are the predictive values of $\alpha, \beta$, and $\lambda ; \widehat{T}_{m}$, $\widehat{T}_{h f}, \widehat{T}_{h r}, \widehat{T}_{m 1}, \widehat{T}_{m 2}$ are predictive values which are obtained by $\widehat{\alpha}, \widehat{\beta}$, and $\widehat{\lambda}$.

4.3. Dynamic Simulation. Generally, for the series system, the optimum braking stability object can be realized easily through the coordination between the generators and 


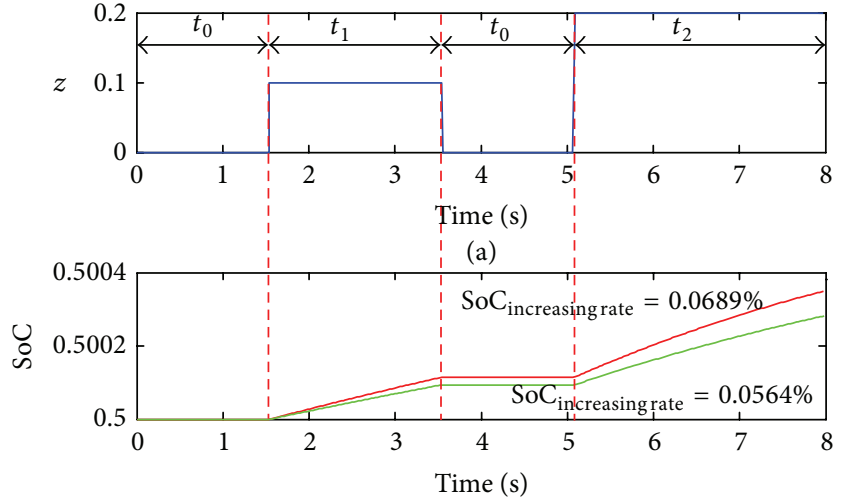

With the predictive distribution model
With the regenerative braking torque
distribution strategy of $1: 1$

(b)

FIgURE 14: The simulation results of SoC.

the hydraulic brakes. So, with regard to the predictive distribution model, the only advantage is embodied in the regenerative braking energy recovery efficiency. To verify the advantages of the predictive distribution model, two simulation models of the vehicle are established in MATLAB/Simulink. One is established with the predictive distribution model, the other is based on the ideal braking force distribution strategy which is also striving to maximize the regenerative braking energy recovery and optimize the braking stability simultaneously. Additionally, no optimization method is used in the other simulation model and the distribution of the regenerative braking torque is based on $1: 1$.

The other braking force distribution strategy can be expressed as follows.

First, based on the requirement of the ideal braking force distribution, the required braking torque of the front and rear wheels under a required braking torque can be obtained as follows:

$$
\begin{aligned}
& T_{\text {front_require }}+T_{\text {rear_require }}=T_{r m}, \\
& \frac{T_{\text {front_require }}}{T_{r m}}=\beta_{\text {opt }},
\end{aligned}
$$

where $T_{\text {front_require, }}, T_{\text {rear_require }}$ denote the required braking torque of the front and rear wheels, respectively.

Then, the optimum charging torque $T_{\mathrm{opt}}$ can be calculated by (7), if $T_{\text {opt }}$ is bigger than $T_{\text {front_require }}$, then make $T_{m}=$ $T_{\text {front_require }} ; T_{h f}=0 ; T_{h r}=T_{\text {rear_require. }}$. If $T_{\text {opt }}$ is lower than or equal to $T_{\text {front_require }}$, then make $T_{m}=T_{\text {opt }} ; T_{h f}=T_{\text {front_require }}-$ $T_{\text {opt }} ; T_{h r}=T_{\text {rear_require }}$.

Finally, the distribution of $T_{m}$ between two generators is $1: 1$.

In the simulation models, the initial vehicle speed is $48 \mathrm{~km} / \mathrm{h}$. The battery SoC is 0.5 . The road is assumed to be a dry pavement. Two cooperative braking processes are defined in Figures 14(a)-16(a); the value of $t_{0}$ is 1.54 , which is the interval time between two braking processes, $t_{1}$ is the first braking time, and $t_{2}$ is the second braking time.

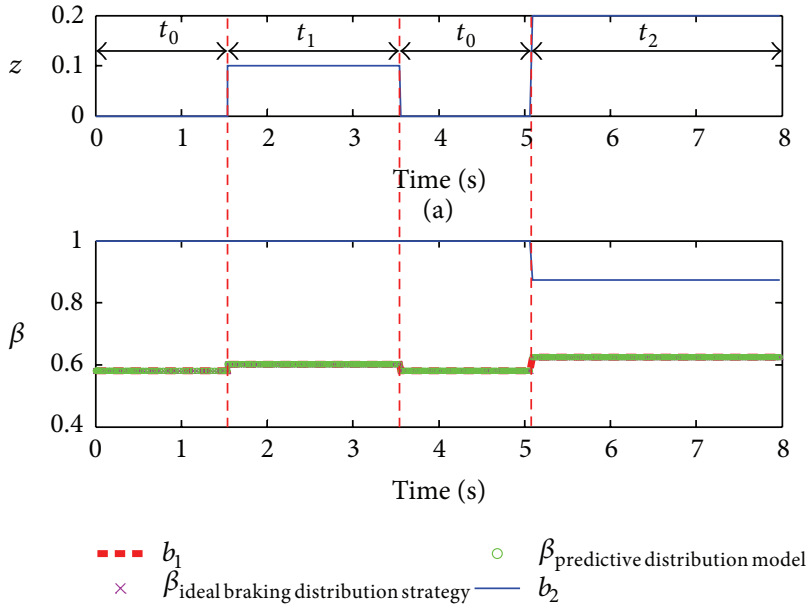

(b)

Figure 15: The simulation results of $\beta$.

Define the increasing rate of $\mathrm{SoC}$ as follows:

$$
\mathrm{SoC}_{\text {increasing rate }}=\frac{\mathrm{SoC}_{\text {end }}-\mathrm{SoC}_{\text {initial }}}{\mathrm{SoC}_{\text {initial }}},
$$

where $\mathrm{SoC}_{\text {increasing rate }}$ denotes the increasing rate of SoC; and $\mathrm{SoC}_{\text {end }}$ denotes the end state of SoC; and $\mathrm{SoC}_{\text {initial }}$ denotes the initial state of SoC.

As shown in Figure 14, during the braking time of $t_{0}$, SoC will remain unchanged for the two models. During the braking time of $t_{1}$, SoC will increase linearly and the advantage of the predictive distribution model begins to manifest, which is that $\mathrm{SoC}_{\text {increasing rate }}$ is bigger than the ideal braking force distribution strategy. During the braking time of $t_{2}$, the increasing tendency is similar to $t_{1}$, and the only difference is that the advantage of $\mathrm{SoC}_{\text {increasing rate }}$ is highlighted in this braking process. The reason can be interpreted to the fact that the bigger $z$ is, the more regenerative braking energy will be obtained and the advantage of the predictive distribution model will be more obvious. At the end of braking, the simulation model with the predictive distribution model $\mathrm{SoC}_{\text {increasing rate }}$ is $0.0689 \%$, the other is $0.0564 \%$.

It can be seen from Figure 15, both of the braking force distribution of the models are close to $b_{1}$, and fall into the scope between $b_{1}$ and $b_{2}$, which means that the vehicle can avoid any wheel be locked earlier, in addition, if the wheels be locked, all the wheels will be locked at the same time for the both simulation models. So the vehicle can maintain the optimum braking stability.

Figure 16 shows the simulation results of the predictive distribution model. If $z$ is 0 , which is corresponding to the braking time of $t_{0}, v$ is almost not changed. $\alpha_{\text {predictive value }}$ and $T$ are equal to 0 , and no braking torque will be generated by the braking apparatuses. $\lambda_{\text {predictive value }}$ is equal to 1 , actually, whatever the value of $\lambda$ is, the ultimate output torque for every braking apparatus will be unaffected, the reason is that $z$ is equal to 0 which is the precondition for the output braking torques. Additionally, the values of $\alpha_{\text {predictive value }}$ and $\lambda_{\text {predictive value }}$ make no sense during this braking time and the 


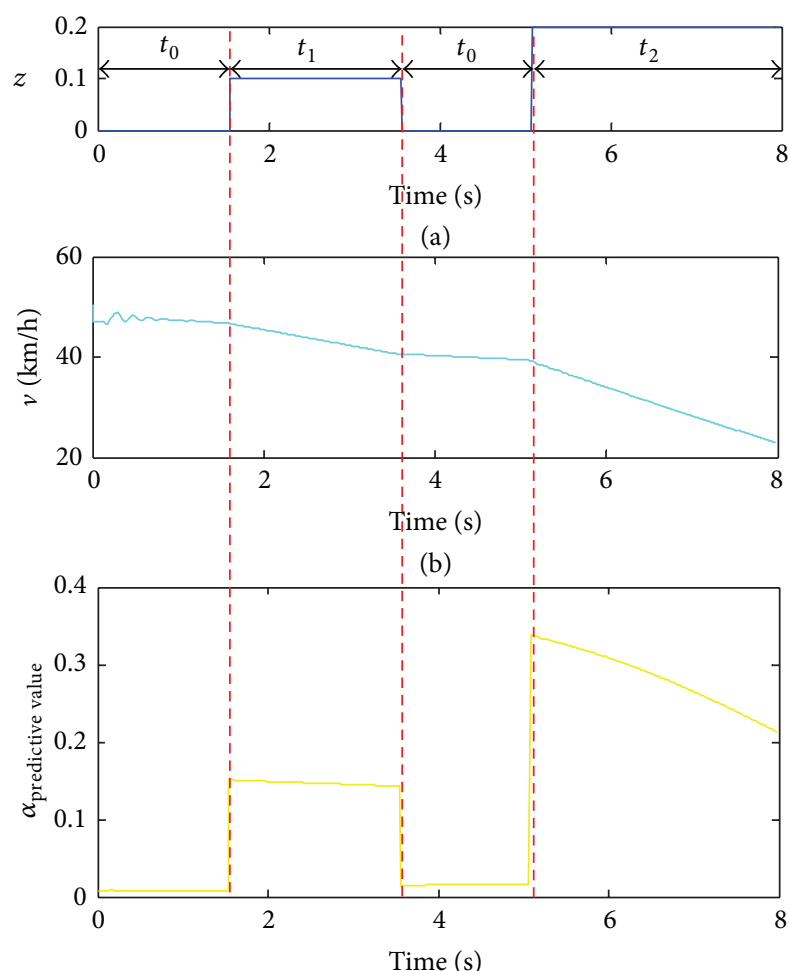

(c)

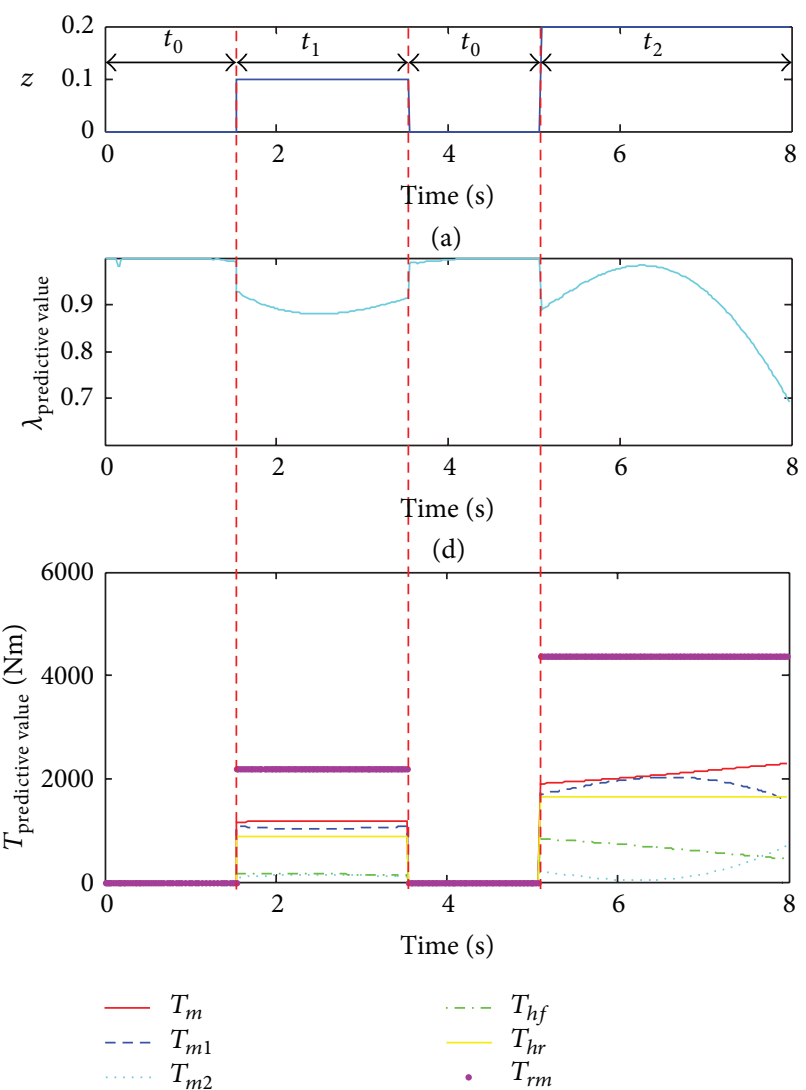

(e)

FIGURE 16: The simulation results of the predictive distribution model.

values are wrong as a result of the design space of $z(0.01-$ 0.4 ). During the braking process of $t_{1}, z$ is 0.1 , which is a relatively low braking severity, so the vehicle decelerates slowly. $\alpha_{\text {predictive value }}$ and $\lambda_{\text {predictive value }}$ are changed and they meet the cooperative braking objects' requirements. $\alpha_{\text {predictive value }}$ changes linearly and $\lambda_{\text {predictive value }}$ changes nonlinearly. Since the value of $\lambda_{\text {predictive value }}$ is close to 1 , generator 1 will afford the mostly charging torque. During the braking time of $t_{2}$, the changing trends of variables are similar to $t_{1}$. The only difference is that the descending slope of $v$ is bigger than $t_{1}$ due to the higher braking severity.

\section{Conclusions}

This paper carries out a systematic study for a predictive distribution model of a series cooperative braking system. Three disciplines of the maximum regenerative energy recovery capability, the maximum generating efficiency, and the optimum braking stability are considered with an off-line optimization method. In consideration of the poor real-time performance of optimization, a predictive model which is based on the off-line optimization data is presented. Finally, a predictive distribution model which is constituted by the predictive model and an additional condition is proposed. The offline optimization data proves that the optimization method can meet every discipline and improve the cooperative braking performance. The dynamic simulation results show that the predictive distribution model is reasonable for realtime control.

\section{Conflict of Interests}

The authors declare that there is no conflict of interests regarding the publication of this paper.

\section{References}

[1] J. Zhang, D. Kong, L. Chen, and X. Chen, "Optimization of control strategy for regenerative braking of an electrified bus equipped with an anti-lock braking system," Journal of Automobile Engineering, vol. 226, no. 4, pp. 494-506, 2012.

[2] J. Zhang, X. Chen, and P.-J. Zhang, "Integrated control of braking energy regeneration and pneumatic anti-lock braking," Journal of Automobile Engineering, vol. 224, no. 5, pp. 587-610, 2010.

[3] C. Jo, J. Ko, H. Yeo, T. Yeo, S. Hwang, and H. Kim, "Cooperative regenerative braking control algorithm for an automatictransmission-based hybrid electric vehicle during a downshift," Journal of Automobile Engineering, vol. 226, no. 4, pp. 457-467, 2012.

[4] J. Zhang, C. Lv, J. Gou, and D. kong, "Cooperative control of regenerative braking and hydraulic braking of an electrified 
passenger car," Journal of Automobile Engineering, vol. 226, no. 10, pp. 1289-1302, 2012.

[5] M. Shang, L. Chu, J. Guo, Y. Fang, and F. Zhou, "Braking force dynamic coordinated control for hybrid electric vehicles," in Proceedings of the 2nd IEEE International Conference on Advanced Computer Control (ICACC '10), vol. 4, pp. 411-416, Shenyang, China, March 2010.

[6] B. Cao, Z. Bai, and W. Zhang, "Research on control for regenerative braking of electric vehicle," in Proceedings of the IEEE International Conference on Vehicular Electronics and Safety, pp. 92-97, Shaan'xi, China, October 2005.

[7] H. Yeo, S. Hwang, and H. Kim, "Regenerative braking algorithm for a hybrid electric vehicle with CVT ratio control," Journal of Automobile Engineering, vol. 220, no. 11, pp. 1589-1600, 2006.

[8] Y. Li, Q. Zeng, C. Wang, and L. Wang, "Research on control strategy for regenerative braking of a plug-in hybrid electric city public bus," in Proceedings of the 2nd International Conference on Intelligent Computing Technology and Automation (ICICTA '09), vol. 1, pp. 842-845, Changsha, China, October 2009.

[9] J. Zhang, D. Ren, B. Song, G. Sun, and G. Sun, "The research of regenerative braking control strategy for advanced braking force distribution," in Proceedings of the 5th International Conference on Natural Computation (ICNC '09), vol. 6, pp. 458462, Tianjin, China, August 2009.

[10] F. Sangtarash, V. Esfahanian, and H. Nehzati, "Effect of different regenerative braking strategies on braking performance and fuel economy in a hybrid electric bus employing CRUISE vehicle simulation," in Proceedings of the SAE International Powertrains, Fuels and Lubricants Congress, 2008.

[11] L. Chu, W. Sun, L. Yao et al., "Integrative control strategy of regenerative and hydraulic braking for hybrid electric car," in Proceedings of the 5th IEEE Vehicle Power and Propulsion Conference (VPPC '09), pp. 1091-1098, Dearborn, Mich, USA, September 2009.

[12] Z. Jingming, S. Baoyu, and N. Xiaojing, "Optimization of parallel regenerative braking control strategy," in Proceedings of the IEEE Vehicle Power and Propulsion Conference (VPPC '08), pp. 1-4, Harbin, China, September 2008.

[13] J. Guo, J. Wang, and B. Cao, "Regenerative braking strategy for electric vehicles," in Proceedings of the IEEE Intelligent Vehicles Symposium, pp. 864-868, Xi'an, China, June 2009.

[14] J. Simard and S. Sarraillon, "Electric vehicle braking system," U.S. Patent Application 10/459, 594, 2003.

[15] M. Ogura, Y. Aoki, and S. Mathison, "The Honda EV PLUS regenerative braking system," in Proceeding of the 14th International Electric Vehicle Symposium, 1997.

[16] Y. Sasaki, A. Otomo, and F. Kawahata, “Toyota braking system for hybrid vehicle with regenerative system," in Proceedings of the 14th International Electric vehicle Symposium and Exhibition, 1997.

[17] J. Ahn, K. Jung, and D. Kim, "Modeling and simulation of electro-mechanical brake for hybrid electric vehicles," in Proceedings of the 22th International Electric Vehicle Symposium and Exhibition, pp. 687-697, 2006.

[18] C. Jo and H. Kim, "Performance analysis of electro-mechanical brake system for a hybrid electric vehicle using HILS," in Proceedings of the 23rd International Electric Vehicle Symposium and Exhibition, pp. 979-988, 2007.

[19] H. Yeo and H. Kim, "Development of regenerative braking control algorithm and electro-hydraulic module for a hybrid electric vehicle," in Proceedings of the 20th International Electric Vehicle Symposium and Exhibition, pp. 3891-3899, 2003. 


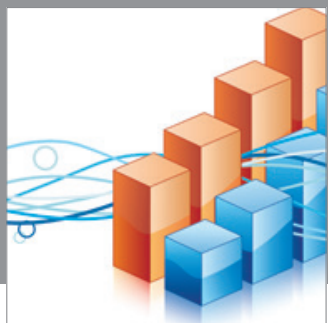

Advances in

Operations Research

mansans

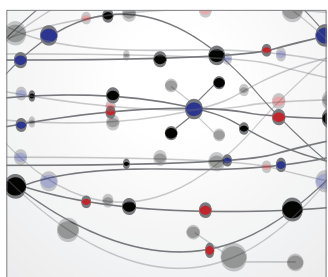

The Scientific World Journal
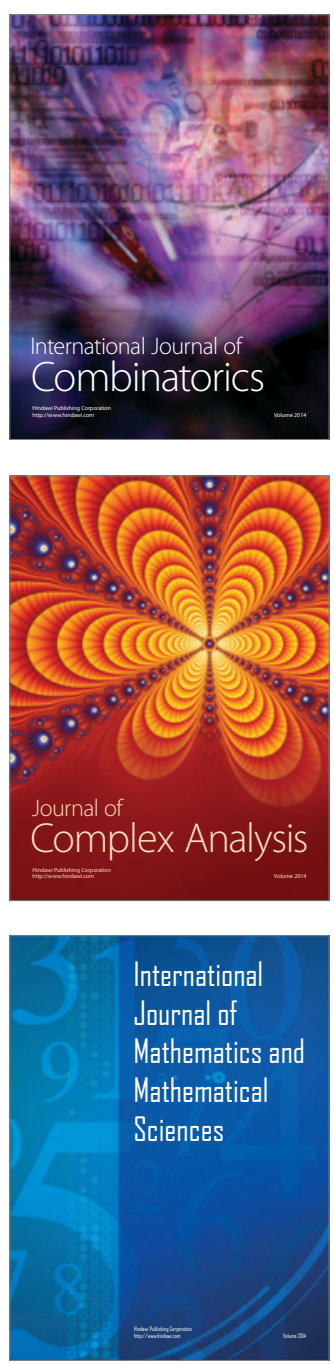
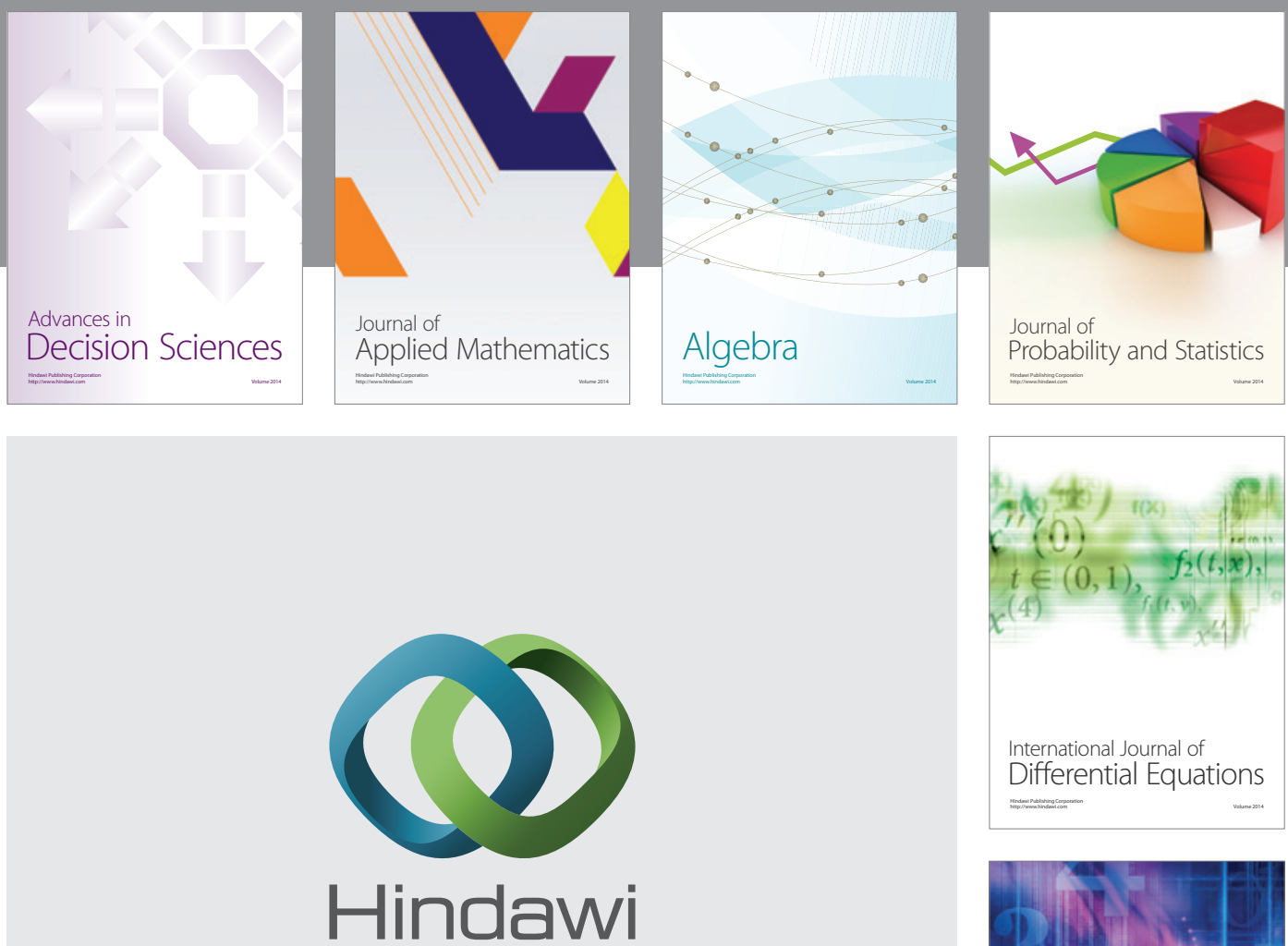

Submit your manuscripts at http://www.hindawi.com
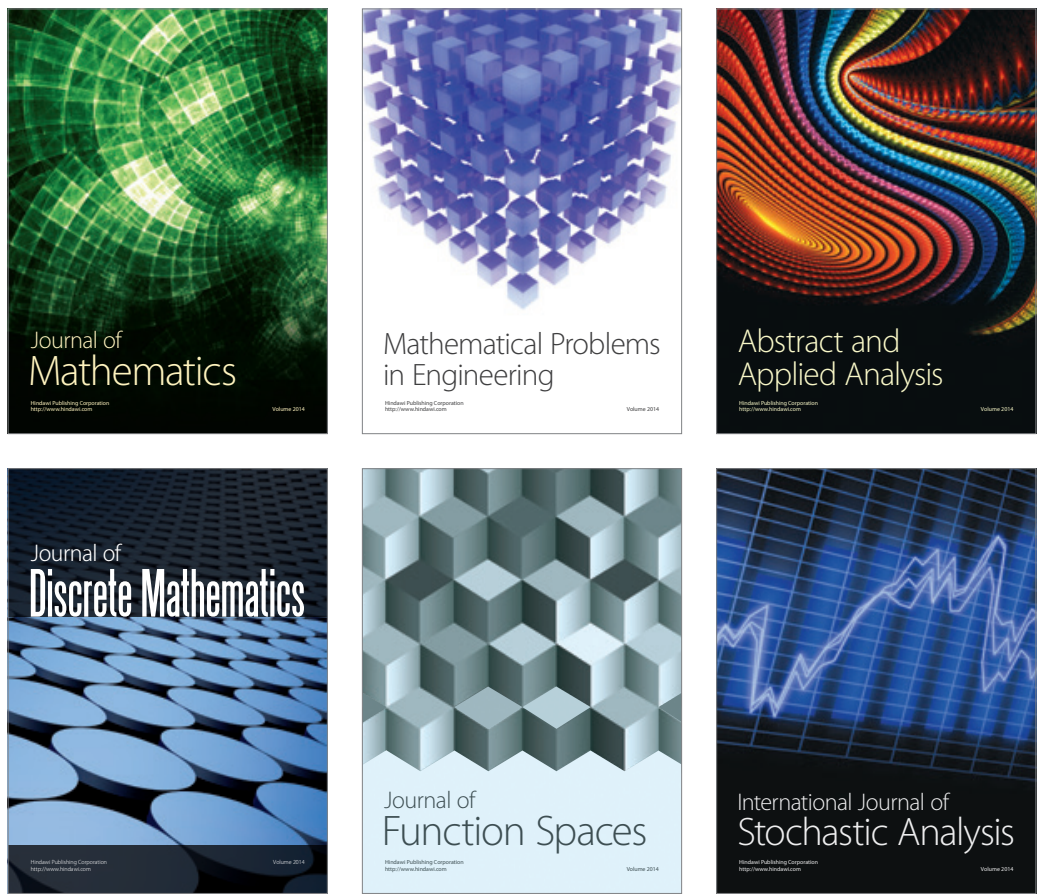

Journal of

Function Spaces

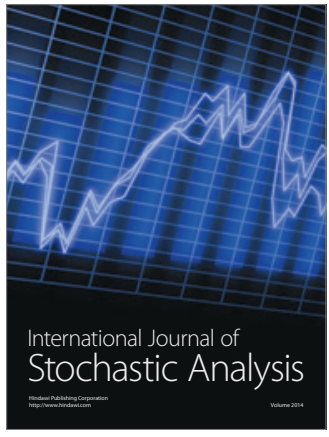

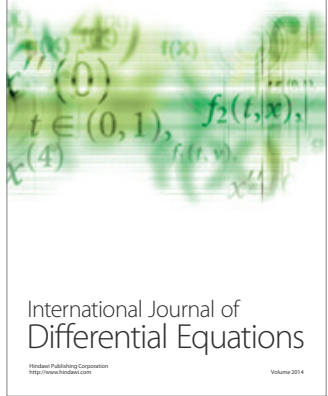
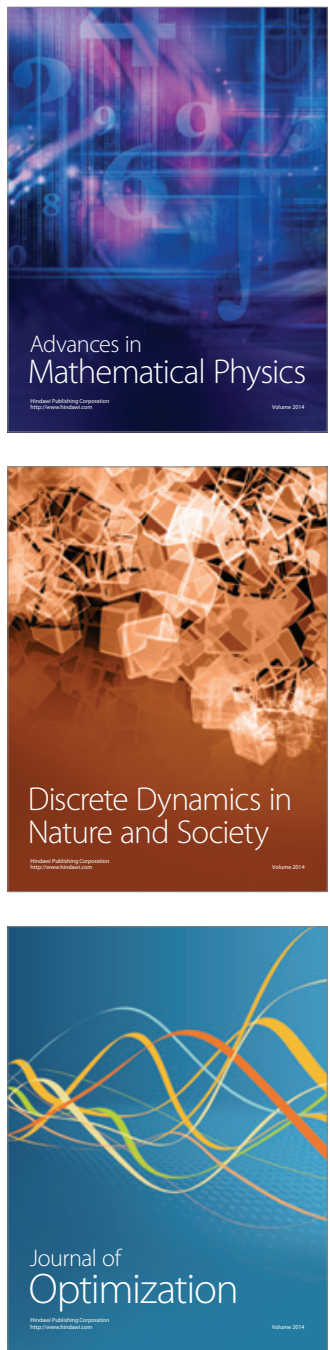